\title{
Haptic wrist guidance using vibrations for Human-Robot teams
}

This is the peer reviewed version of the following article:

Original:

Aggravi, M., Salvietti, G., Prattichizzo, D. (2016). Haptic wrist guidance using vibrations for Human-Robot teams. In Proc. 2016 25th IEEE International Symposium on Robot and Human Interactive Communication (RO-MAN) (pp.113-118). New York : IEEE [10.1109/ROMAN.2016.7745098].

Availability:

This version is availablehttp://hdl.handle.net/11365/993326

since 2017-05-24T19:16:14Z

Publisher:

IEEE

Published:

DOI:10.1109/ROMAN.2016.7745098

Terms of use:

Open Access

The terms and conditions for the reuse of this version of the manuscript are specified in the publishing policy. Works made available under a Creative Commons license can be used according to the terms and conditions of said license.

For all terms of use and more information see the publisher's website.

(Article begins on next page) 


\title{
Haptic Wrist Guidance Using Vibrations for Human-Robot Teams
}

\author{
Marco Aggravi ${ }^{1}$, Gionata Salvietti ${ }^{1,2}$, and Domenico Prattichizzo ${ }^{1,2}$
}

\begin{abstract}
Human-Robot teams can efficiently operate in several scenarios including Urban Search and Rescue (USAR). Robots can access areas too small or deep for a person, can begin surveying larger areas that people are not permitted to enter and can carry sensors and instruments. One important aspect in this cooperative framework is the way robots and humans can communicate during rescue operation. Vision and audio modalities may result not efficient in case of reduced visibility or high noise. A promising way to guarantee effective communications between robot and human in a team is the exploitation of haptic signals. In this work, we present a possible solution to let a robot guide the position of a human operator's hand by using vibrations. We demonstrate that an armband embedding four vibrating motors is enough to guide the wrist of an operator along a predefined path or in a target location. The results proposed can be exploited in human-robot teams. For instance, when the robot detects the position of a sensible target, it can guide the wrist of the operator in such position following an optimal path.
\end{abstract}

\section{INTRODUCTION}

Haptic guidance refers to the possibility of guiding the whole body or a part of it using haptic stimuli [1]. Haptic guidance has been used in different applications, both to display directional indications while walking (see, e.g., [2], [3]) or to guide selected motions of a part of the body, see e.g., [4]. Target locations are usually predefined and the system takes care of tracking the human body and of displaying directional cues through haptic interfaces.

Between the several solutions proposed, an interesting scenario is when a robot guides a human. In [5], for instance, an autonomous, interactive tour-guide robot is presented. Beside this possible application for a robot guiding a human, a novel use of haptic feedback for human navigation with a mobile robot has been presented in [6]. Assuming that a path-planner has provided a mobile robot with an obstaclefree trajectory, the vehicle could steer the human from an initial to a desired target position by only interacting with him/her via a custom-designed vibrotactile bracelet. The arising cooperative navigation system resulted not intrusive, flexible, and easy-to-use. In [7] the system was extended to a possible use in urban search and rescue (USAR). In particular, authors investigated how a robot can guide a human agent using a vibrotactile bracelet in a reduced visibility scenario. The robot was able to compute its own path and was also able to track the position of the human agent using a dedicated sensor, i.e., a RGB-D camera. Robots may be

The research leading to these results has received funding from the European Union under grant agreement n. 601165 of the project "WEARHAP WEARable HAPtics for humans and robots" and under grant agreement $\mathrm{n}$. 643644 of the project "ACANTO: A CyberphysicAl social NeTwOrk using robot friends"

${ }^{1}$ Marco Aggravi, Gionata Salvietti, and Domenico Prattichizzo are with the Department of Information Engineering and Mathematics, University of Siena, Via Roma 56, I-53100 Siena, Italy.

${ }^{2}$ Gionata Salvietti and Domenico Prattichizzo are also with the Department of Advanced Robotics, Istituto Italiano di Tecnologia, via Morego 30, I-16163 Genoa, Italy

\{aggravi, salviettigio, prattichizzo\}@dii.unisi.it

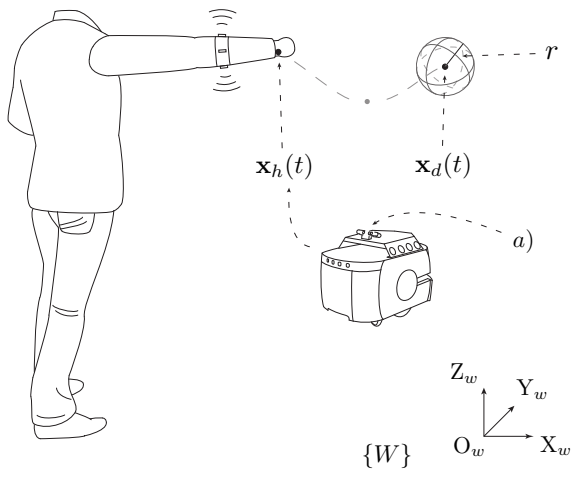

Fig. 1. Haptic wrist guidance using vibrations for human-robot teams: the users wrist $x_{h}$ is guided, via vibrotactile cues, toward a desired position, represented by a sphere of radius $r$ centered in $x_{d}$. A robot, here represented by a mobile robot, is in charge of deciding the desired position for the user's wrist, and of tracking the human body/arm with a camera (a)).

able to enter disaster sites which are otherwise too dangerous or too difficult for humans to get to. Once there, robots can gather information about the situation, providing human operators with video feeds, maps, and sensor data. Using this information, humans might be able to make a better situation assessment, to aid emergency management [8]. Referring to the scenario depicted in Fig. 1, a robot could be able to recognize an important target (e.g., a valve, a switch, etc.) thanks to its on board sensors and guide the human toward it. As an example, in USAR the robot could guide a firefighter inside a building and then guide the operator's wrist/hand toward a valve following a predefined trajectory that avoid crossing high temperature areas revealed by a temperature sensor. We suppose that the robot is able to compute its own path. The path can be computed localizing itself on an available map of the area or building a new map using the on-board sensors. Moreover, the final path can be selected according also to other sensors' measurements, e.g., temperature, percentage of oxygen, etc.. The robot is also able to track the position of the human agent using a dedicated sensor, e.g., a RGB-D camera.

In this paper, we focus on a guiding system where a vibrating bracelet is used to guide the wrist - and thus the hand-in a certain position and to let the wrist follow a predefined path/trajectory. For directional guidance inside a building, the reader is referred to [6], [7].

During the last years, vibrotactile devices have not only been used to support visually impaired users, but there are several applications, in which these devices are also applied for assisting sighted persons [9], [10]. In particular, attempt of enhancing the spatial awareness of human operators has gained an increasing attention. In [11] and [12], a vibrotactile feedback device was evaluated for spatial guidance in a tracking task paradigm. Users had to translate and rotate 
virtual objects according to the vibrotactile vs. verbal cues without visual information. In [13], a tactile stimulation device was added in a stroke rehabilitation system that uses a teaching robot. An interesting novel approach for guidance tasks is that described in [14]. In this work, the authors exploited electrical muscle stimulations (EMS) and vibrations for a 3D virtual hand pointing task. The authors performed an experimental validation, in which they compared no feedback, visual cues, EMS, and vibrating suggestions. Results demonstrated that both EMS and vibration provided reasonable addition to visual feedback.

In this work, we take advantage of a haptic armband with only four vibrating motors. Different vibration patterns are used to guide the human wrist motion. The use of a single armband guarantees high wearability and portability of the system, while reducing power consumption and increasing the total autonomy. The proposed framework has been tested with seven subjects in two different tasks. In the former, we evaluated the capability of the system to guide the human wrist in an exact location. In the latter, we evaluated how the system can display a predefined path to the user. To prove the reliability of the guidance with a vibrating armband, we use an external tracking system instead of a camera on-board the robot.

The rest of the paper is organized as follows. Sect. II presents our haptic guidance system, detailing its realization and solved issues. In Sect. III, we validate the proposed system via real-world experiments, whereas in Sect. IV, results are given and discussed. Eventually, in Sect. V, we summarize the main contributions of the paper, and we discuss possible directions for future research.

\section{Haptic Guidance System}

In the previous section, the problem was introduced, and state-of-the-art solutions were discussed. We here describe the novelty of our system, and its realization.

\section{A. System Description}

Referring to Fig. 1, let $\{W\}=\left\langle\mathrm{O}_{w}, \mathrm{X}_{w}, \mathrm{Y}_{w}, \mathrm{Z}_{w}\right\rangle$ be the world reference frame, representing the origin and the main axes of a generic 3D Cartesian space. In what follows, variables and quantities will be expressed w.r.t. such world reference frame $\{W\}$. Let $\mathbf{x}(t)=[x(t), y(t), z(t)]^{T} \in \mathbb{R}^{3}$ be a generic point, at time $t \in \mathbb{R}_{>0}$, and let $\{H\}(t)=$ $\left\langle\mathbf{x}(t), \mathrm{X}_{w}, \mathrm{Y}_{w}, \mathrm{Z}_{w}\right\rangle$ be the reference frame associated to that point. In addition, we associate to each point $\mathbf{x}(t)$ a plane $\mathscr{P}(t)$, which is the plane spanned by $\mathrm{X}_{w}$ and $\mathrm{Z}_{w}$ axis of $\{H\}(t)$ (see Fig. 2).

Referring to Fig. 2, let $\mathbf{x}_{h}(t)=\left[x_{h}(t), y_{h}(t), z_{h}(t)\right]^{T} \in$ $\mathbb{R}^{3}$ be the position of the user's wrist at time $t \in \mathbb{R}_{>0}$, with its associated reference frame $\left\{H_{h}\right\}(t)$ and plane $\mathscr{P}_{h}(t)$. Moreover, let $\mathbf{x}_{d}(t)=\left[x_{d}(t), y_{d}(t), z_{d}(t)\right]^{T} \in \mathbb{R}^{3}$ be a desired reference for the wrist at time $t \in \mathbb{R}_{>0}$, with its associated reference frame $\left\{H_{d}\right\}(t)$ and plane $\mathscr{P}_{d}(t)$. Eventually, let us define $\mathbf{p}(t) \in \mathbb{R}^{2}$ as the orthogonal projection of $\mathbf{x}_{h}(t)$ onto the desired plane $\mathscr{P}_{d}(t)$.

With these definitions, we have that the distance error between the user's wrist and its desired position can be expressed as

$$
\mathbf{e}(t)=\left[\begin{array}{c}
e_{l}(t) \\
\mathbf{e}_{p}(t)
\end{array}\right]
$$

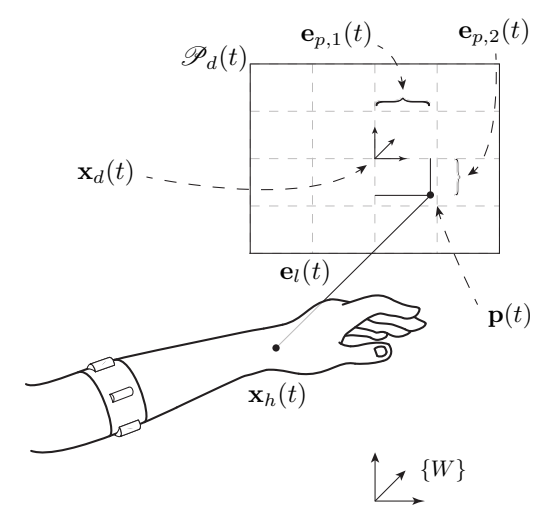

Fig. 2. The user's wrist position $\mathbf{x}_{h}(t)$ is projected onto the plane $\mathscr{P}_{d}(t)$, resulting in $\mathbf{p}(t)$. The distance between $\mathbf{x}_{h}(t)$ and $\mathbf{p}(t)$ along $\mathrm{Y}$ of $\{W\}$ is $e_{l}(t)$, while the distance between $\mathbf{p}(t)$ and the desired position $\mathbf{x}_{d}(t)$ on $\mathscr{P}(t)$ is $\mathbf{e}_{p}$.

being $e_{l}(t)=y_{h}(t)-y_{d}(t)$ the distance between $\mathscr{P}_{h}(t)$ and $\mathscr{P}_{d}(t)$ along $\mathrm{Y}_{w}$, and

$$
\mathbf{e}_{p}(t)=\left[\begin{array}{c}
e_{p, 1}(t) \\
e_{p, 2}(t)
\end{array}\right]=\left[\begin{array}{c}
x_{h}(t)-x_{d}(t) \\
z_{h}(t)-z_{d}(t)
\end{array}\right]
$$

The final aim of our guidance system is to guide the user's wrist toward and into a sphere of radius $r \in \Re_{>0}$, centered in $\mathbf{x}_{d}\left(t_{e}\right)$ (see Fig. 1), at any time $t_{e} \in \mathbb{R}_{>0}$, i.e., the ending time of the trial. In other words, if the user's wrist has an Euclidean norm of the distance error lower than a certain threshold $r$, i.e., $\left\|\mathbf{e}\left(t_{e}\right)\right\|_{2}<r$, we consider our approach as working.

\section{B. Hardware implementation}

In order to guide the user's wrist, we provide vibrotactile stimuli via a haptic wristband. The device comprises four Precision Microdrives 307-100 Pico Vibe 9mm vibration motors [15] — with a vibration frequency range of 100$280 \mathrm{~Hz}$, an Arduino Mini Pro $3.3 \mathrm{~V}$, a $3.7 \mathrm{~V}$ LiPo battery, and a RN-42 Bluetooth 2.1 antenna. The electronics and the battery are embedded into a 3D-printed case, while the vibrating motors are enclosed by the producer in waterproof containers. During the evaluation, a 3D printed support was mounted on the top of the electronics case, for holding retroreflective passive markers, used for the optical tracking of the device. The device and its components are shown in Fig. 3(a) and 3(b).

\section{Guidance policy}

Differently from [11], [12], where only planar movements were considered, and from [13], where a longitudinal motion was suggested with additional motors, we focused on obtaining a 3D guidance system with one band, comprising four vibrating motors, as previously described. Such motors were placed so that they would span the four main movements directions on a plane, i.e., up (U), left (L), down (D), and right (R) direction (see Fig. 3(c)). Two of the motors (U and D) were also used to convey haptic suggestions along the direction normal to the plane on which the four motors lie. Each motor $j \in\{\mathrm{U}=1, \ldots, \mathrm{R}=4\}$ is independently controllable, both in vibration frequency $f_{j}(t) \in[100-280] \mathrm{Hz}$ 


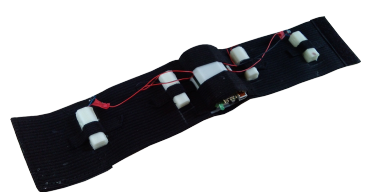

(a)

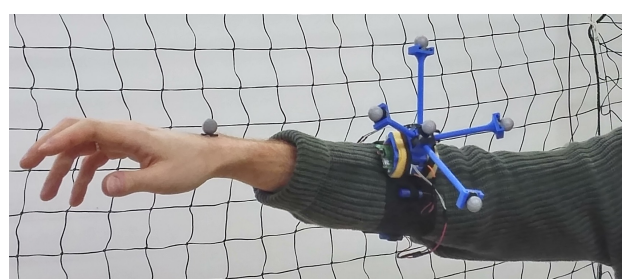

(b)

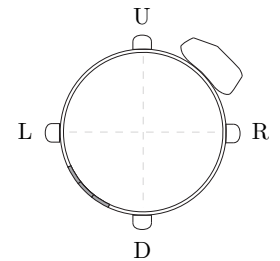

(c)

Fig. 3. Vibrotactile haptic devices: (a) proposed device; (b) proposed device worn by one subject; (c) vertical section of the proposed device, with the four motors (U, R, D, and L) depicted. In (c), the user's wrist is supposed to be entering the figure.

and in vibration interval $i_{j}(t)=k \tau \mathrm{s}$, being $\tau$ the minimal vibration burst and $k \in \mathbb{N}$.

Vibration frequencies were selected to avoid possible on/off behaviors. We set different thresholds for different vibration frequencies, based on the distance from the desired point, and on the human perception [16]. In [17], the authors evaluated the vibrotactile perception of relatively young users with a device similar to that used in this work. They found that in a range $80-200 \mathrm{~Hz}$, a frequency variation of around $30 \mathrm{~Hz}$ was perceived. Acknowledging this variation as the $25 \%$ of the total range of the investigated frequencies, we defined four levels of vibration thresholds, i.e., $\mathscr{T}^{f}=$ $\left\{t h_{1}^{f}, \ldots, t h_{4}^{f}\right\}$ related to four distance thresholds $\mathscr{T}^{d}=$ $\left\{t h_{1}^{d}, \ldots, t h_{4}^{d}\right\}$. The vibration interval was selected to have different timings so that a same motor could be exploited for more than one direction suggestions. In particular, the up (U) and down motors (D) were chosen to convey two intervals of vibration: a "fast" vibration $i_{1,3}(t)=\tau \mathrm{s}$, related to distance between the actual plane $\mathscr{P}_{h}(t)$ and the desired plane $\mathscr{P}_{d}(t)$, i.e., $e_{l}(t)$, and a "slow" vibration $i_{1,3}(t)=2 \tau$ s. This interval vibration difference was related to the distance on the plane $\mathscr{P}_{d}(t)$ of $\mathbf{p}(t)$, as explained in the following section. The vibration interval for motors $\mathrm{L}$ and $\mathrm{R}$ was fixed to $i_{2,4}(t)=2 \tau$ s with no variations.

Let us now describe the algorithm we propose for obtaining haptic suggestions. In what follows, we drop the dependency on time $t \in \mathbb{R}_{>0}$, for the sake of simplicity. It is worth to note that the following policy is applied to every instant of time during a trial. Let us consider a desired wrist position $\mathbf{x}_{d}$, an actual wrist position $\mathbf{x}_{h}$ and its projection p onto $\mathscr{P}_{d}$, as described in Sect. II-A. Moreover, let us consider $s(\cdot)$ as the sign function

$$
s(x)=\left\{\begin{array}{ll}
-1 & \text { if } x<0 \\
0 & \text { if } x=0 \\
1 & \text { if } x>0
\end{array}, x \in \mathbb{R} .\right.
$$

From the projection of $\mathbf{x}_{h}$ onto $\mathscr{P}_{d}$ we have a distance error $\mathbf{e}=\left[e_{l}, \mathbf{e}_{p}\right]^{T}$, which will be used to obtain the vibration frequencies. As a first step, we check if the distance $e_{l}$ between the two planes is-in absolute value-inside the distance thresholds

$$
f^{l}= \begin{cases}t h_{4}^{f} & \text { if }\left|e_{l}\right| \geq t h_{4}^{d} \\ t h_{3}^{f} & \text { if } t h_{4}^{d}>\left|e_{l}\right| \geq t h_{3}^{d} \\ t h_{2}^{f} & \text { if } t h_{3}^{d}>\left|e_{l}\right| \geq t h_{2}^{d} \\ t h_{1}^{f} & \text { if } t h_{2}^{d}>\left|e_{l}\right| \geq t h_{1}^{d} \\ 0 & \text { if } t h_{1}^{d}>\left|e_{l}\right|\end{cases}
$$

This step defines the vibration frequency for the guidance along $\mathrm{Y}_{w}$, i.e., guidance toward the plane $\mathscr{P}_{d}$. Consequently, we check if the distance on the plane $\mathscr{P}_{d}$ is-in absolute value-inside the distance thresholds

$$
f_{k}^{p}= \begin{cases}t h_{4}^{f} & \text { if }\left|e_{p, k}\right| \geq t h_{4}^{d} \\ t h_{3}^{f} & \text { if } t h_{4}^{d}>\left|e_{p, k}\right| \geq t h_{3}^{d} \\ t h_{2}^{f} & \text { if } t h_{3}^{d}>\left|e_{p, k}\right| \geq t h_{2}^{d} \\ t h_{1}^{f} & \text { if } t h_{2}^{d}>\left|e_{p, k}\right| \geq t h_{1}^{d} \\ 0 & \text { if } t h_{1}^{d}>\left|e_{p, k}\right|\end{cases}
$$

being $k \in\{1,2\}$, which provides the vibration frequencies for the guidance along $\mathrm{X}_{w}$ and $\mathrm{Z}_{w}$, i.e., guidance on the plane $\mathscr{P}_{d}$. Once we have obtained $f^{l}$ and $f_{i}^{p}$, we need to check the signs of the distances $e_{l}$ and $\mathbf{e}_{p}$, i.e., the directions to which we need to guide the wrist to. For selecting $f_{2}$ and $f_{4}$, i.e., the vibration frequencies for $\mathrm{R}$ and $\mathrm{L}$, respectively, we check the sign of $e_{p, 1}$ as

$$
f_{2}=\left\{\begin{array}{ll}
f_{1}^{p} & \text { if } s\left(e_{p, 1}\right)>0 \\
0 & \text { else }
\end{array}, f_{4}=\left\{\begin{array}{ll}
f_{1}^{p} & \text { if } s\left(e_{p, 1}\right)<0 \\
0 & \text { else }
\end{array} .\right.\right.
$$

These definitions, together with the followings, model an attractive haptic feedback procedure. For instance, a vibration on $\mathrm{L}$ means "move the wrist toward left". For selecting $f_{1}$ and $f_{3}$, the vibration frequencies for $\mathrm{U}$ and $\mathrm{D}$, respectively, the sign of $e_{l}$ is first checked so to recognize if a motion of the wrist toward the plane $\mathscr{P}_{d}$ is needed. Then, the sign of $e_{p, 2}$ is checked so to understand if up and down movements are needed. These two checks also lead to the different vibration interval $i_{1}$ and $i_{3}$ that are necessary to distinguish the guidance directions. From Equations (1) and (2) the frequencies associated to $\mathrm{U}$ or $\mathrm{D}$ could be $f^{l}$ and $f_{2}^{p}$ at the same time. In this case, we assigned a high priority to the guidance of the user's wrist toward the plane $\mathscr{P}_{d}$. Then the wrist is guided toward the goal position $\mathbf{x}_{d}$. For this reason, the following policy is adopted for choosing $f_{1}$ or $f_{3}$

$$
\begin{aligned}
& f_{1}= \begin{cases}f^{l} & \text { if }\left(s\left(e_{l}\right)>0 \& \& s\left(e_{p, 2}\right)>0\right) \| \\
& \left(s\left(e_{l}\right)>0 \& \& s\left(e_{p, 2}<0\right) \& \& f^{l} \geq f_{2}^{p}\right) \\
f_{2}^{p} & \text { if }\left(s\left(e_{l}\right)<0 \& \& s\left(e_{p, 2}\right)<0\right) \| \\
& \left(s\left(e_{l}\right)>0 \& \& s\left(e_{p, 2}<0\right) \& \& f^{l}<f_{2}^{p}\right) \\
0 & \text { else }\end{cases} \\
& f_{3}= \begin{cases}f^{l} & \text { if }\left(s\left(e_{l}\right)<0 \& \& s\left(e_{p, 2}\right)<0\right) \| \\
f_{2}^{p} & \text { if }\left(s\left(e_{l}\right)>0 \& \& s\left(e_{p, 2}>0\right) \& \& f^{l} \geq f_{2}^{p}\right) \\
0 & \left(s\left(e_{l}\right)<0 \& \& s\left(e_{p, 2}>0\right) \& \& f^{l}<f_{2}^{p}\right) \\
0 & \text { else }\end{cases}
\end{aligned}
$$




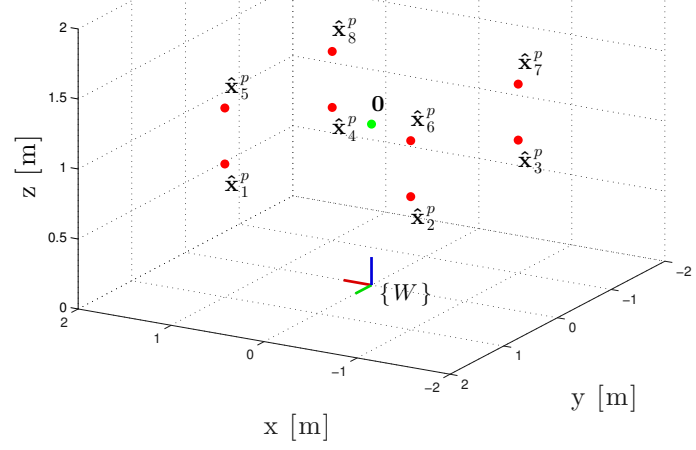

Fig. 4. Point-to-point guidance scenario Desired wrist positions $\hat{\mathbf{x}}_{k}^{p}$, center of the parallelepiped figure $\mathbf{0}$ (green dot), and world reference frame $\{W\}$.

TABLE I

SETTINGS FOR THE PERFORMED EXPERIMENTAL VALIDATION

\begin{tabular}{cl}
\hline \hline $\mathscr{T}^{f}$ & $\left\{t h_{1}^{f}, t h_{2}^{f}, t h_{3}^{f}, t h_{4}^{f}\right\}=\{80,110,140,170\} \mathrm{Hz}$ \\
$\mathscr{T}^{d}$ & $\left\{t h_{1}^{d}, t h_{2}^{d}, t h_{3}^{d}, t h_{4}^{d}\right\}=\{0.1,0.15,0.25,0.4\} \mathrm{m}$ \\
$r$ & $0.141 \mathrm{~m}$ \\
$\tau$ & $0.2 \mathrm{~s}$
\end{tabular}

Moreover, to produce two guidance modalities which are clearly distinguishable, i.e., guidance toward the plane and guidance on the plane toward the desired position, we selected the vibration interval for $\mathrm{U}$ and $\mathrm{D}$ as

$$
i_{1}=\left\{\begin{array}{ll}
\tau & \text { if } f_{1}=f^{l} \\
2 \tau & \text { if } f_{1}=f_{2}^{p}
\end{array}, \quad i_{3}=\left\{\begin{array}{ll}
\tau & \text { if } f_{3}=f^{l} \\
2 \tau & \text { if } f_{3}=f_{2}^{p}
\end{array} .\right.\right.
$$

Eventually, the four motors $j \in\{1, \ldots, 4\}$ of the haptic armband are activated at vibration frequencies $f_{j}$ and vibration interval $i_{j}$.

Remark: The aforementioned checks for $\mathrm{U}$ and $\mathrm{D}$ are done independently, so it is possible that both $\mathrm{U}$ and D motors vibrate, but with different timing and frequencies.

\section{EXPERIMENTAL VALIDATION}

The experimental validation was performed on seven healthy subjects (four males, age range 21-45, four right-handed). Five of them had no previous experience with vibrotactile interfaces. None of the participants reported any deficiencies in perception abilities or physical impairments. The participants signed informed consent forms, and were informed about the purpose of the experiment, being able to discontinue participation at any time.

We considered two possible guiding scenarios for evaluating our system: point-to-point $(\mathrm{P})$ and trajectory guidance $(\mathrm{T})$. In the former, participants were asked to follow the vibration cues until they place their wrist inside a sphere with radius $r \in \Re_{>0}$ centered in $\mathbf{x}_{d}$ (see Fig. 1). Eight points-belonging to $3 \mathrm{D}$ space having $\{W\}$ as origin-were selected as the desired positions for the users' wrists. These eight points $\mathbf{x}_{d}=\hat{\mathbf{x}}_{k}^{p}, k \in\{1, \ldots, 8\}$ were the sharp edges of a parallelepiped, whose center lied at $\mathbf{0}=[0,0,1.15]^{T}$ and with size $2 \times 2 \times 0.4 \mathrm{~m}(\mathrm{~L} \times \mathrm{W} \times \mathrm{H})$. The center of the parallelepiped is shown in Fig. 4, while the eight desired positions are depicted with red spheres in Fig. 4 and with black sphere in Fig. 5(a)-(h).
The desired positions were arranged in a pseudo-random order, whose list was the same for all the participants.

For each trial, the user was asked to start standing still above the origin of $\{W\}$, until the testing attempt was started at $t=0$. The user was wearing the vibrating armband around her/his right arm, placed as in Fig. 3(b). After this moment, the user was provided with haptic suggestions, whose vibration frequency decreased when reducing the distance between the actual wrist position and the desired position (see Sect. II-C). The trial was considered over when the user's wrist distance $\mathbf{x}_{h}\left(t_{e}\right)$ was such that $\left\|\mathbf{e}\left(t_{e}\right)\right\|_{2}<r$, with $t_{e}>t$, being $\mathbf{e}\left(t_{e}\right)=\mathbf{x}_{h}\left(t_{e}\right)-\hat{\mathbf{x}}^{p}$.

For what concerns the trajectory guidance, participants were asked to move their wrist along a 3-D path, composed of multiple desired positions $\mathbf{x}_{k}^{s}, k \in \mathbb{N}$, reaching each time the inside of a sphere of radius $r \in \Re_{>0}$ having as center the correspondent $\mathbf{x}_{k}^{s}$. A eight-shape figure was chosen as a paradigmatic $3 \mathrm{D}$ path. The figure was composed by 15 desired positions, composing the set $\mathbb{X}=\left\{\hat{\mathbf{x}}_{1}^{s}, \ldots, \hat{\mathbf{x}}_{15}^{s}\right\}$, ordered so that the eight-shape figure was covered for two times, starting from $\hat{\mathbf{x}}_{1}$ (namely "1" in Fig. 6(a)) and finishing in $\hat{\mathbf{x}}_{15}$ (namely "15" in Fig. 6(a)). Fig. 6(a) shows the order of the desired positions required to the users. The trial was considered over when the user's wrist distance $\mathbf{x}_{h}\left(t_{e}\right)$ was such that $\left\|\mathbf{e}\left(t_{e}\right)\right\|_{2}<r$, with $t_{e}>t$, being $\mathbf{e}\left(t_{e}\right)=\mathbf{x}_{h}\left(t_{e}\right)-\hat{\mathbf{x}}_{15}^{s}$.

Remark: Since the user is given with no haptic suggestion as soon as the wrist position enters the sphere of radius $r$ around $\mathbf{x}_{d}$, if two consequent points in a path are too close they can be considered as one. In particular, if the two desired consequent positions have an Euclidean distance lower than $2 r$, there is an overlap of the two spheres, thus producing a zone in which the user's wrist is not commanded to the next position. This aspect has to be taken into account when sampling the trajectory for the wrist.

Each participant performed 32 trials for scenario P (four repetitions for eight desired wrist positions) and 4 trials for scenario $\mathrm{T}$, thus resulting in 252 collected trials. No time requirements were designed for the both experimental modalities. For each trial, the user's wrist position was acquired with an optical tracking system (Vicon Motion Systems Ltd, Oxford, UK), composed of eight cameras. The haptic vibrating armband was equipped with five passive retro-reflective optical markers located as in Fig. 3(b). A sixth marker was placed in correspondence of the actual user's wrist, so that it was possible to obtain the wrist position from the haptic device position. The six markers are visible in Fig. 3(b).

The settings values for the evaluation are summarized in Table I. For choosing such parameters, we did consider the evaluation of a similar haptic device as presented in [18] and in [19]. In [18] (Section 4.3.2), the authors found that a JND of $25 \%$ could be perceived as intensity change for around $80 \%$ of the subjects testing similar vibrotactile bracelets. For this reason, we did use a similar percentage for obtaining our thresholds for the distance error. Moreover, in [19], the authors found that the minimal vibration burst that can be perceived by users was that of duration $\tau=0.2 \mathrm{~s}$ using vibrating bracelets. For this reason, we did employ this duration for the fast vibration patterns, whereas we did use a double duration, thus very distinguishable, for the slow 


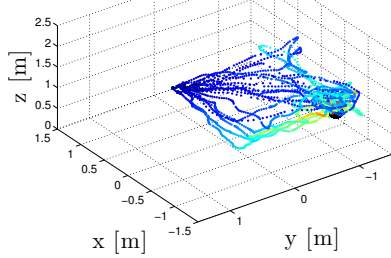

(a)

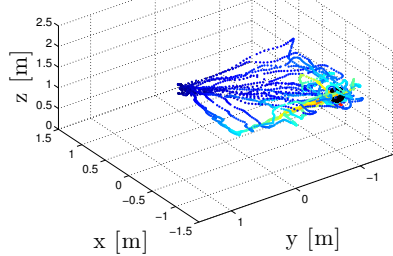

(e)

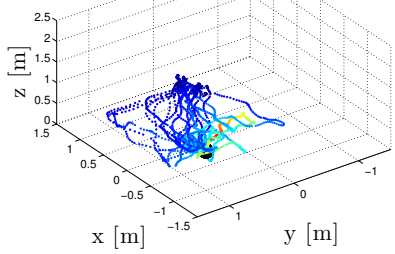

(b)

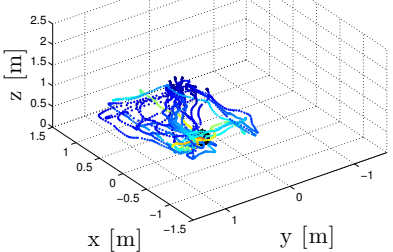

(f)

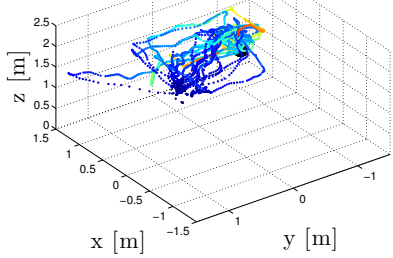

(c)

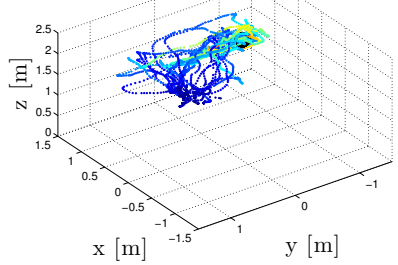

(g)

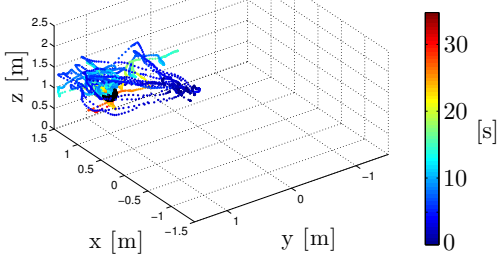

(d)

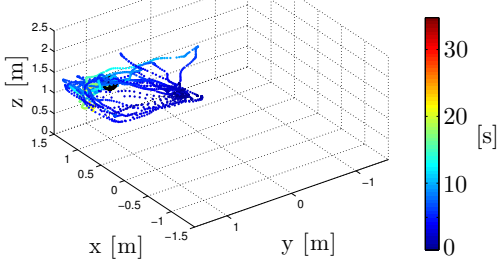

(h)

Fig. 5. Point-to-point guidance scenario: (a)-(h) desired positions for the point-to-point guidance scenario (black sphere) and performed trials for the seven participants. Colors are shown in the colorbars, and they depict the time elapsing during the trial, going from the beginning (blue) to the end of the it (red). In the plots, it is possible to see that the users were following the haptic suggestions by following the priority we chose for the vibrating cues, thus firstly reaching the plane of $\mathbf{x}_{h}(t)$ and then moving on this plane for reaching the desired point.

TABLE II

MEAN COMPLETION TIME (AND STANDARD DEVIATION) FOR EACH DESIRED POSITION $\hat{\mathbf{x}}_{k}^{p}, k \in\{1, \ldots, 8\}$ AND FOR THE 3D PATH POSITIONS $\mathbb{X}$.

\begin{tabular}{cccc}
$\hat{\mathbf{x}}_{k}^{p}$ & time [s] & $\hat{\mathbf{x}}_{k}^{p}$ & time [s] \\
\hline \hline$\hat{\mathbf{x}}_{1}^{p}$ & $16.98 \pm 6.93$ & $\hat{\mathbf{x}}_{5}^{p}$ & $10.42 \pm 5.70$ \\
$\hat{\mathbf{x}}_{2}^{p}$ & $15.61 \pm 5.98$ & $\hat{\mathbf{x}}_{6}^{p}$ & $12.85 \pm 5.91$ \\
$\hat{\mathbf{x}}_{3}^{p}$ & $18.31 \pm 6.33$ & $\hat{\mathbf{x}}_{7}^{p}$ & $15.77 \pm 7.22$ \\
$\hat{\mathbf{x}}_{4}^{p}$ & $17.43 \pm 6.46$ & $\hat{\mathbf{x}}_{8}^{p}$ & $15.99 \pm 5.92$ \\
\hline & time $[\mathrm{s}]$ & & \\
$\mathbb{X}$ & $156.38 \pm 36.63$ &
\end{tabular}

vibration patterns. Distances $\mathscr{T}^{d}$ as well as the radius $r$ of target spheres have been experimentally evaluated. We performed several trials varying the thresholds and the radius from smaller to bigger. The values reported in this work are those presenting the best results in terms of mean completion time. We did not report other results with different thresholds for the sake of brevity.

At the end of each session of trials, a NASA Task Load Index (TLX) questionnaire [20] was propose to the participant, with the aim of assessing the perceived workload in terms of Mental Demand (MD), Temporal Demand (TD), Physical Demand (PD), Performance (PR), Effort (EF), and Frustration (FR). Each of the six questions of the questionnaire has a scale of 21 levels, considering 1 as "very low" and 21 as "very high".

\section{RESUlts AND Discussion}

In this section, we describe and discuss the results of our experimental validation.

Point-to-point guidance: Each of the participant was able to reach the eight desired wrist positions. We considered as evaluation parameter the time it took to the participants to reach the destination points, while following the haptic guiding cues provided by our system. Fig. 5 shows the trajectories performed by the seven participants to our evaluation, for each desired wrist position (depicted with a black spheres). Time to complete each trial can be evaluated considering the colorbar in Fig. 5. The first two rows of Table II resume the mean completion time for each desired wrist position $\hat{\mathbf{x}}_{k}^{p}$. From Table II, we can see that the completion time was higher for the points placed at lower height w.r.t. to $\mathbf{0}$, i.e., $\hat{\mathbf{x}}_{1, \ldots, 4}^{p}$, than for points placed at higher height w.r.t. to $\mathbf{0}$, $\hat{\mathbf{x}}_{5, \ldots, 8}^{p}$. This was expected since the starting initial position for all the participants was closer to $\hat{\mathbf{x}}_{5, \ldots, 8}^{p}$ than to $\hat{\mathbf{x}}_{1, \ldots, 4}^{p}$.

Trajectory guidance: All participants were able to complete the task. Fig. 6 shows one particular trial, among the 28 recorded. In particular, Fig. 6(b)-(c)-(d) shows the user's wrist positions from top view, rear view, and side view, respectively. The timing of the trial is shown with colors, ranging from blue (beginning of the trial) to red (end of the trial). Fig. 6(a) shows the order of the desired wrist positions $\hat{\mathbf{x}}_{k}^{s}, k \in\{1, \ldots, 15\}$. The last column of Table II resumes the mean completion time for the eight-shape desired wrist positions.

For what concerns the perceived workload demanded by our guiding system, the raw question results of the TLX questionnaires are depicted in Table III. From the Table, it is possible to see that the frustration (FR) in using our system was very low, together with the effort (EF) it demanded. Concerning the performance (PR), the users perceived a good success rate in completing the task (here the lower the score

TABLE III

MEAN RAW SCORE AND STANDARD DEVIATION FOR THE NASA-TLX WORKLOAD QUESTIONNAIRE.

\begin{tabular}{ccccccc} 
& MD & PD & TD & PR & EF & FR \\
\hline \hline mean raw score & 7.14 & 8.14 & 8.14 & 5.42 & 5.57 & 4.14 \\
standard deviation & 2.60 & 2.96 & 5.08 & 3.25 & 1.39 & 2.34
\end{tabular}




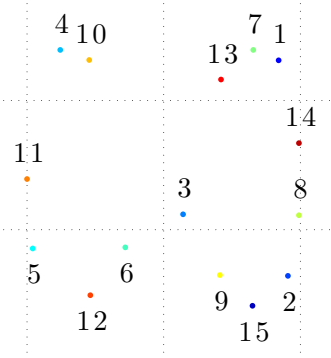

(a)

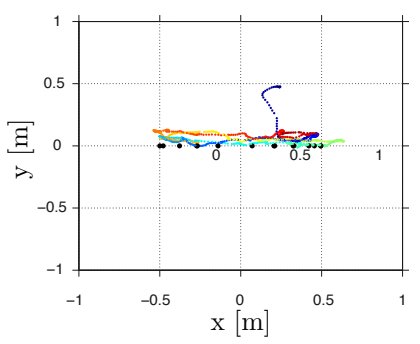

(b)

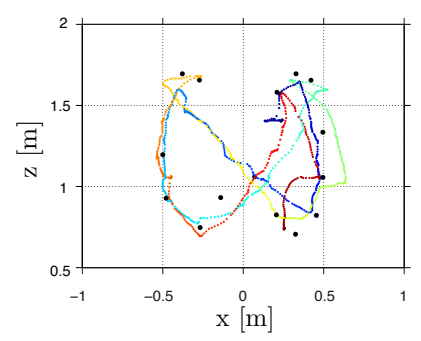

(c)

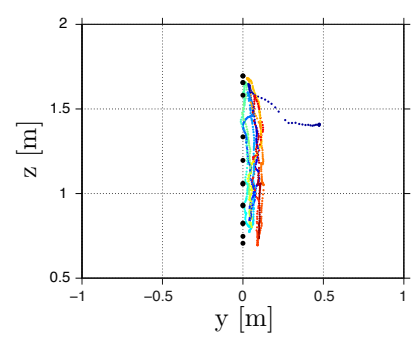

(d)

Fig. 6. $3 D$ path guidance scenario: (a) Order of the desired positions $\hat{\mathbf{x}}_{k} \in \mathbb{X}, k \in\{1, \ldots, 15\}$, for the eight-shape figure and example of a trial performed by user number 2, from (b) top view, (c) rear view, and (d) side view. (b)-(d) Position of the user's wrist during the trial (blue to red dots); (b)-(d) desired positions for the 3D path guidance scenario (black sphere), arranged as a eight-shape figure. It is possible to see the high priority that the user took in firstly moving towards the plane on which $\hat{\mathbf{x}}_{1}$ lies, and then by moving on such plane reaching one-by-one the other points of the path.

the better the perception of success). The mental, physical, and temporal demands (MD, PD, and TD, respectively) scored a bit higher than the other metrics, but we can consider such values acceptable, considering the properties of our guiding system.

\section{CONCLUSION}

In this work we present a possible framework to guide the user wrist position using a vibrating armband. In particular we used only four motors to perform two different tasks in a three-dimensional environment. All participant were able to fulfill the proposed tasks. The proposed system can be exploited in human-robot teams. The haptic communication channel can be used an effective way to let a robots display a target position or a desired path to a human mate. The same system could be also used for assistive guidance for blind people. Suppose a visual impaired user taking advantage of the mobile robot as a guide in a supermarket. Once the robot has guided the user in front of a shelf, the robot can guide the wrist in the exact product location.

We are currently integrating the tracking system in a mobile robot. We are also investigating solutions to suggest the orientation of the wrist in addition to the 3-D position. Possible improvements are envisioned also with the inclusion of the wrist's DoFs into the haptic suggestions computation algorithm.

\section{REFERENCES}

[1] D. Feygin, M. Keehner, and F. Tendick, "Haptic guidance: Experimental evaluation of a haptic training method for a perceptual motor skill," in Haptic Interfaces for Virtual Environment and Teleoperator Systems, 2002. HAPTICS 2002. Proceedings. 10th Symposium on. IEEE, 2002, pp. $40-47$.

[2] S. Bosman, B. Groenendaal, J.-W. Findlater, T. Visser, M. de Graaf, and P. Markopoulos, "Gentleguide: An exploration of haptic output for indoors pedestrian guidance," in Human-computer interaction with mobile devices and services. Springer, 2003, pp. 358-362.

[3] S. Shoval, I. Ulrich, and J. Borenstein, "NavBelt and the Guide-Cane [obstacle-avoidance systems for the blind and visually impaired]," Robotics \& Automation Magazine, IEEE, vol. 10, no. 1, pp. 9-20, 2003

[4] A. Stanley and K. Kuchenbecker, "Evaluation of Tactile Feedback Methods for Wrist Rotation Guidance," IEEE Transactions on Haptics, vol. 5, no. 3, pp. 240-251, 2012.

[5] W. Burgard, A. B. Cremers, D. Fox, D. Hähnel, G. Lakemeyer, D. Schulz, W. Steiner, and S. Thrun, "Experiences with an interactive museum tour-guide robot," Artificial intelligence, vol. 114, no. 1, pp. 3-55, 1999.
[6] S. Scheggi, M. Aggravi, F. Morbidi, and D. Prattichizzo, "Cooperative human-robot haptic navigation," in Proceedings IEEE International Conference on Robotics and Automation, ICRA, no. 0, Hong Kong, China, 2014, pp. 2693-2698.

[7] S. Scheggi and G. Salvietti, "Haptic guidance in urban search and rescue scenarios with reduced visibility," in Proc. 20th IMEKO TC4 Int. Symp. and 18th Int. Workshop on ADC Modelling and Testing Research on Electric and Electronic Measurement for the Economic Upturn, Benevento, Italy, 2014.

[8] G.-J. M. Kruijff, F. Colas, T. Svoboda, J. Van Diggelen, P. Balmer, F. Pirri, and R. Worst, "Designing intelligent robots for human-robot teaming in urban search and rescue." in Proceedings of the AAAI 2012 Spring Symposium on Designing Intelligent Robots, 2012.

[9] T. Oron-Gilad, J. L. Downs, R. D. Gilson, and P. A. Hancock, "Vibrotactile guidance cues for target acquisition," Systems, Man, and Cybernetics, Part C: Applications and Reviews, IEEE Transactions on, vol. 37, no. 5, pp. 993-1004, 2007.

[10] V. Lehtinen, A. Oulasvirta, A. Salovaara, and P. Nurmi, "Dynamic tactile guidance for visual search tasks," in Proceedings of the 25th annual ACM symposium on User interface software and technology. ACM, 2012, pp. 445-452.

[11] B. Weber, S. Schätzle, T. Hulin, C. Preusche, and B. Deml, "Evaluation of a vibrotactile feedback device for spatial guidance," in World Haptics Conference (WHC), 2011 IEEE. IEEE, 2011, pp. 349-354.

[12] S. Schätzle, T. Ende, T. Wüsthoff, and C. Preusche, "Vibrotac: An ergonomic and versatile usable vibrotactile feedback device," in Proceedings IEEE International Symposium on Robot and Human Interactive Communication, ROMAN. IEEE, 2010, pp. 670-675.

[13] Y. Jin, H. Chu, E. Kim, and S. Kang, "VT-ware: A wearable tactile device for upper extremity motion guidance," in Proceedings IEEE International Symposium on Robot and Human Interactive Communication, ROMAN, 2014, pp. 335-340.

[14] M. Pfeiffer and W. Stuerzlinger, " $3 \mathrm{~d}$ virtual hand pointing with ems and vibration feedback," in 3D User Interfaces (3DUI), 2015 IEEE Symposium on. IEEE, 2015, pp. 117-120.

[15] Precision Microdrives, https://catalog.precisionmicrodrives.com/orderparts/product/303-100-3mm-vibration-motor-8mm-type, Feb. 2016.

[16] A. Riener, Sensor Actuator Supported Implicit Interaction in Driver Assistance Systems. Springer, 2010.

[17] S. Scheggi, F. Chinello, and D. Prattichizzo, "Vibrotactile haptic feedback for human-robot interaction in leader-follower tasks," in Proceedings of the 5th International Conference on PErvasive Technologies Related to Assistive Environments. ACM, 2012, pp. 51-54.

[18] S. Scheggi, F. Morbidi, and D. Prattichizzo, "Human-robot formation control via visual and vibrotactile haptic feedback," IEEE Transactions on Haptics, vol. 7, no. 4, pp. 499-511, 2014.

[19] S. Scheggi, M. Aggravi, and D. Prattichizzo, "A vibrotactile bracelet to improve the navigation of older adults in large and crowded environments," in Proc. 20th IMEKO TC4 Int. Symp. and 18th Int. Workshop on ADC Modelling and Testing Research on Electric and Electronic Measurement for the Economic Upturn, 2014.

[20] S. G. Hart, "NASA-task load index (NASA-TLX); 20 years later," in Proceedings of the human factors and ergonomics society annual meeting, vol. 50, no. 9. Sage Publications, 2006, pp. 904-908. 